\title{
Idiopathic dilatation of pulmonary artery: a case report
}

\author{
Authors: Sara Soraja Nasri ${ }^{1}$, Narcisse Nasri ${ }^{1}$, Gordana Pavliša ${ }^{1,2}$ (mentor) \\ ${ }^{1}$ School of Medicine, University of Zagreb, Zagreb, Croatia \\ ${ }^{2}$ Department for Respiratory Diseases Jordanovac Zagreb, University Hospital Center Zagreb, Zagreb, \\ Croatia
}

DOI: https://doi.org/10.26800/LV-142-supp5-15

\section{Introduction:}

Idiopathic dilatation of pulmonary arteries (IDPA) is an uncommon condition with an abnormality of pulmonary arteries, it is believed to be a benign anomaly. The incidence is $0.6 \%$ in patients with congenital heart disease. IDPA was recognised as a clinical entity in 1923 by Wessler and Jaches. Even though this condition is well known for almost a century, not much is known about its etiopathology and pathophysiology. Diagnosis is set by excluding all congenital or acquired heart lessons that can lead to dilatation of pulmonary arteries. A long period of follow-up is of great importance as in some patients the underlying pathology is discovered later in life.

\section{Case report:}

We report the case of a 67-year-old female patient who was admitted to our hospital in 2016. because of progressive dyspnea and chest pain (NYHA II). Examination with computer tomography (CT) angiography and echocardiography showed pulmonary regurgitation and heavy dilatation of truncus pulmonalis (in main diameter $58 \mathrm{~cm}$ ), right and left pulmonary artery $(33 \mathrm{~mm} / 40 \mathrm{~mm}$ ), as well as dilatation of right ventricular with no sign of hypertrophy. Echocardiography did not show signs of pulmonary hypertension. By right heart catheterization (RHC) pulmonary hypertension (mPAP $24 \mathrm{mmHg}$ ) was not confirmed. Radiocardiography showed left to right shunt of $13 \%$ and scintigraphy with Tc-99m did not show possible thromboembolic lung disease. She was diagnosed with IDPA. On re-evaluation in October of 2019 her functional and radiologic status was without significant change.

\section{Conclusion:}

In conclusion, pressure of $24 \mathrm{mmHg}$ shown by right ventricular catheterization and $13 \%$ of right to left shunt does not explain the massive dilatation of pulmonary arteries. In addition, there are no significant changes in status of this patient in 3 years of follow-up thus this confirms the diagnosis of IDPA. Even though long-time follow-up is crucial to establish its benign course.

Keywords: benign anomaly, idiopathic dilatation of pulmonary artery, truncus pulmonalis 